Check for updates

Cite this: RSC Adv., 2019, 9, 38257

Received 13th October 2019

Accepted 8th November 2019

DOI: $10.1039 / c 9 r a 08354 \mathrm{e}$

rsc.li/rsc-advances

\section{Different phase-dominated chiral assembly of polyfluorenes induced by chiral solvation: axial and supramolecular chirality $\dagger$}

\begin{abstract}
Shuai Li, ${ }^{a}$ Tengfei Miao, ${ }^{a}$ Xiaoxiao Cheng, ${ }^{a}$ Yin Zhao, ${ }^{a}$ Wei Zhang (D) *a and Xiulin Zhu ${ }^{\text {ab }}$
The introduction of chirality in an achiral system will not only help avoid the tedious and expensive synthesis of chiral substances or catalysts but also greatly expand the ranges of chiral compounds. Herein, the induction of chirality in achiral polyfluorene (PF2/6 and PF8) with different alkyl chains at the C9 position of fluorene was achieved using a binary solvent system, in which ethanol was used as a poor solvent and chiral limonene was employed simultaneously as a good solvent and chiral solvent. The circular dichroism (CD), UV-vis and photoluminescence (PL) spectra demonstrated that the structures of PFs with linear/branched alkyl side chains and the volume fractions of the cosolvents had an obvious effect on the generation of chirality driven by chiral solvation. During the chiral assembly processes of PFs, PF8 with a linear alkyl side chain demonstrated the obvious chiral $\beta$ phase, while PF2/6 with a branched alkyl side chain only showed the chiral $\alpha$ phase. WAXD data also confirmed the existence of quite different phases of PF8 and PF2/6. The first induced chirality of PF with a branched alkyl side chain (PF2/6) will help the further understanding of the chiral assembly mechanism of PFs driven by chiral solvation. The induced chirality of PF2/6 was axial chirality of the PF chain but the chirality of PF 8 was from the supramolecular chiral assembly of the PF chains. The linear dependence of the maximum CD and $g_{C D}$ values on the enantiomeric purity of chiral limonene demonstrated that the achiral PFs have a potential application as chiral sensors to detect the ee value of limonene.
\end{abstract}

\section{Introduction}

As one of the most important structural characteristics of life, much attention has been paid to design chiroptical materials at a molecular to supramolecular level by a self-assembly strategy. ${ }^{1}$ Generally speaking, the chirality of supramolecular systems is generated by the assembly of chiral components through noncovalent interactions, such as hydrogen bonding, ${ }^{2} \pi-\pi$ stacking, ${ }^{3,4}$ and van der Waals interaction. ${ }^{5}$ Meanwhile, achiral building blocks such as small molecules, oligomers and polymers have been employed to produce chiral supramolecules with the help of circularly polarized light (CPL), ${ }^{6,7}$ asymmetric liquid crystal field, ${ }^{\mathbf{8}, 9}$ chiral solvent, ${ }^{\mathbf{1 0 - 1 3}}$ gelation ${ }^{\mathbf{1 4 , 1 5}}$ and other chiral sources. ${ }^{\mathbf{1 6 - 1 8}}$ Compared with the former, the latter strategy can help avoid the tedious and expensive design and synthesis

${ }^{a}$ Suzhou Key Laboratory of Macromolecular Design and Precision Synthesis, Jiangsu Key Laboratory of Advanced Functional Polymer Design and Application, State and Local Joint Engineering Laboratory for Novel Functional Polymeric Materials, College of Chemistry, Chemical Engineering and Materials Science, Soochow University, Suzhou Industrial Park, Suzhou 215123, China. E-mail: weizhang@suda. edu.cn

${ }^{b}$ Global Institute of Software Technology, No 5. Qingshan Road, Suzhou National HiTech District, Suzhou 215163, China

$\dagger$ Electronic supplementary information (ESI) available. See DOI: $10.1039 / \mathrm{c} 9 \mathrm{ra} 08354 \mathrm{e}$ of chiral substances or catalysts. Chiral solvation has been proven to be an efficient and versatile protocol for inducing chirality in achiral systems, including achiral small molecules, ${ }^{\mathbf{1 9}}$ oligomers $^{2}$ and polymers. ${ }^{20-22}$ In contrast to other chiral solvents, chiral limonene is a good candidate for a chiral source because it is harmless, commercially available, cheap and efficient. Würthner et al. reported the chiral limonene-induced supramolecular chiral assembly of achiral perylene bisimide (PBI) dyes. ${ }^{23}$ Chiral limonene was first employed to induce chirality in syndiotactic polystyrene ( $s$-PS) by Guerra et al. ${ }^{24}$ Recently, limonene was used as an efficient chiral solvent to induce supramolecular chirality in achiral $\pi$-conjugated polymers including linear polyfluorene (PF) derivatives, ${ }^{25-28}$ poly(diphenylacetylene)s (PDPAs) ${ }^{29}$ and hyperbranched $\mathrm{PFs}^{30}$ and further in achiral side chain azobenzene-containing polymer systems. ${ }^{22,31-33}$

The promising optoelectronic properties of chiral $\pi$-conjugated polymers have attracted considerable interests due to the potential applications in circularly polarized light (CPL) emission, ${ }^{34-37}$ chiral sensors ${ }^{38}$ and chiroptical switch materials. ${ }^{39}$ Among them, chiral polyfluorenes (PFs) have been intensely studied due to their excellent thermal, chemical and photochemical stabilities and high fluorescence quantum efficiency benefiting from their rigid structures. ${ }^{36,37,40,41}$ The induction of chirality in achiral PFs has been achieved by means of CPL, ${ }^{\mathbf{4 2 , 4 3}}$ 
chiral solvents ${ }^{25-28,44}$ and chiral polymers. ${ }^{45}$ It is noteworthy that the process of the formation of chiral PFs is often accompanied by chain conformation transformation. Nakano and coworkers first reported the CPL-induced chirality of PFs, in which the existence of the $\beta$ phase was the key factor. For example, the lower-molar-mass poly(9,9-dioctylfluorene-2,7-diyl) (poly(DOF)) and poly(9,9-dihexylfluorene-2,7-diyl) (poly(DHF)) without the $\beta$ phase could not respond to CPL irradiation, but the highermolar-mass poly(DOF) with the $\beta$ phase could respond to CPL irradiation. ${ }^{43}$ The importance of $\beta$ phase for chiral solventinduced chirality of PFs was also reported by our group. ${ }^{21,27,44}$ The CD intensity of PF8 aggregate gradually increased with the progressively propagated $\beta$ phase on prolonging the cooling time. The chirality could not be generated from achiral PFs driven by chiral limonene both in ternary solvent system (good solvent/chiral solvent/poor solvent) and neat limonene if no $\beta$ phase formed in aggregate state. Actually, the $\alpha$ phase indicates that the dihedral angle between adjacent fluorene units is $135^{\circ}$, while the $\beta$ phase describes a much more coplanar structure with a torsion angle of $165^{\circ} .{ }^{46}$ The backbone of $\beta$ phase has a high degree of order, which results in a longer effective conjugation length. ${ }^{47}$ The different properties of $\alpha$ and $\beta$ phase may result in different chiral assembly mechanisms. Therefore, it is significant to study the limonene-induced chiral assembly behaviour of PFs dominated by different phases.

In our previous work, PF derivatives with different linear side chains could self-assemble to form chiral supramolecular structures induced by limonene at low temperature, which was controlled by the odd-even effect of side alkyl chains. ${ }^{44}$ However, PF2/6, with branched side alkyl chains, could not perform chiral assembly in similar conditions, but it could be induced to adopt a preferred-handed helicity in the achiral/ chiral polyfluorene blended thin film. ${ }^{48}$ In this contribution, we first present the different phase-dominated chiral induction and generation in achiral PFs (PF2/6 and PF8) with different mechanisms. In the binary solvent (chiral limonene as a good solvent and chiral solvent, ethanol as a poor solvent) system, the obvious CD signals were observed in the $\alpha$ phase region for PF2/ 6 aggregates, while in the $\alpha$ and $\beta$ phase region for PF8 aggregates. The chirality of PF2/6 aggregates is derived from the axial chirality of the twisted fluorene units in the PF chain, but supramolecular chirality with PF chains as the building blocks is responsible for the chirality of PF8 aggregates. The successful induction of chirality in achiral PF2/6 will produce the circularly polarized electroluminescence material, which is commonly generated from chiral PFs. ${ }^{36,37}$

\section{Experimental section}

Materials

Polyfluorenes (PF2/6 and PF8, Fig. S1 $\dagger$ ) were designed and synthesized by the typical Yamamoto coupling polymerization or Kumada catalyst transfer polycondensation (KCTP) according to previous literature. ${ }^{\mathbf{4 4}}$ The GPC curves and molecular mass characteristics of PF2/6 and PF8 are shown in Fig. S1 and Table $\mathrm{S} 1 . \dagger(R)-(+)$-Limonene $\left(1 R, \mathrm{TCI},>95.0 \%,[\alpha]_{589}^{24}=+99.62^{\circ}\right)$ and $(S)-(-)$-limonene $\left(1 S\right.$, TCI, $\left.>95.0 \%,[\alpha]_{589}^{24}=-97.72^{\circ}\right)$ were used without further purification. All the other chemicals were obtained from Shanghai Development Co., Ltd (Shanghai, China) and used as received.

\section{Preparation of optically active PF aggregates}

Solutions of PFs $\left(3.0 \times 10^{-3} \mathrm{~mol} \mathrm{~L}^{-1}\right)$ in neat $1 R$ or $1 S$ were prepared previously in a $20 \mathrm{~mL}$ flask equipped with a stopcock by refluxing with vigorous stirring at $90{ }^{\circ} \mathrm{C}$ for $1 \mathrm{~h}$ to ensure the complete dissolution of polymers. After cooling to room temperature, the solutions were further filtered through 0.45 $\mu \mathrm{m}$ PTFE filters to remove the dust particles. A $0.05 \mathrm{~mL} \mathrm{PF} /$ limonene solution was transferred to a SQ-cuvette. After that, a certain volume of limonene of $1 S$ or $1 R$ and $\mathrm{EtOH}$ were added. The aggregates were obtained after slight shaking. The other polymer aggregates were produced in a similar way.

\section{Preparation of WAXD samples}

The PF aggregates prepared in the binary solvent system with a volume fraction of $1 / 2(1 S$ or $1 R / \mathrm{EtOH})$ were transferred to glass plates by drop-coating and dried in vacuum for $12 \mathrm{~h}$ at $60{ }^{\circ} \mathrm{C}$.

\section{Measurements}

The molar mass and polydispersity $\left(\nexists=M_{\mathrm{w}} / M_{\mathrm{n}}\right)$ of the polymer were determined using a TOSOH HLC-8320 size exclusion chromatograph (SEC) (Tokyo, Japan) equipped with refractive index and UV detectors using two TSKgel Super Multipore HZ-N columns $(4.6 \times 150 \mathrm{~mm}, 3 \mu \mathrm{m}$ particle size $)$ enabling molecular weight analysis ranging from $7 \times 10^{2}$ to $2 \times 10^{5} \mathrm{~g} \mathrm{~mol}^{-1}$. THF was used as the eluent at a flow rate of $0.35 \mathrm{~mL} \mathrm{~min}^{-1}$ at $40{ }^{\circ} \mathrm{C}$. The samples were calibrated with polystyrene standards (TOSOH). The CD spectra were measured on a JASCO (HachiojiTokyo, Japan) J-815 spectropolarimeter at $25{ }^{\circ} \mathrm{C}$, using a SQgrade cuvette, a bandwidth of $2 \mathrm{~nm}$, a scanning rate of 200 $\mathrm{nm} \min ^{-1}$, a response time of $2 \mathrm{~s}$, a single accumulation, and a path length of $10 \mathrm{~mm}$. The UV-vis spectra were recorded on a Shimadzu UV-3150 spectrophotometer (Shimadzu, Japan). The photoluminescence (PL) spectra were recorded on a PerkinElmer LS-50B spectrofluorometer with $370 \mathrm{~nm}$ as the excitation wavelength at $25{ }^{\circ} \mathrm{C}$. The WAXD data were obtained on an X'Pert-Pro MPD X-ray powder diffractometer (Panalytical, Holland) equipped with a copper target, at a scan speed of $0.375151^{\circ} \mathrm{s}^{-1}$ and at a scattering angle range of $2 \theta=5-40^{\circ}$. Transmission electron microscopy (TEM) images were taken with a Hitachi HT7700 operated at an accelerating voltage of $120 \mathrm{kV}$. The absorption dissymmetry ratio, $g_{\mathrm{CD}}$, is defined as $2\left(A_{\mathrm{L}}-A_{\mathrm{R}}\right) /\left(A_{\mathrm{L}}+A_{\mathrm{R}}\right)$, where $A_{\mathrm{L}}$ and $A_{\mathrm{R}}$ are the absorbance of left and right circularly polarized light, respectively. After transformation, $g_{\mathrm{CD}}=[\theta] /(32980 \times \mathrm{Abs})$.

\section{Results and discussion}

\section{Effect of side alkyl chain structures in PFs on chiral assembly behaviour}

Our previous study demonstrated that PF8 could efficiently selfassemble into a chiral supramolecular structure induced by 
neat limonene at low temperature $\left(-10{ }^{\circ} \mathrm{C}\right.$ to $\left.-30{ }^{\circ} \mathrm{C}\right)$, but $\mathrm{PF} 2 / 6$ with branched side chain did not respond to limonene chirality even at a much lower temperature $\left(-80{ }^{\circ} \mathrm{C}\right) .{ }^{44}$ It was noted that $\beta$ phase played a key important role in supramolecular chiral assembly of PFs induced by limonene chiral solvation. In order to realize the chiral solvation-induced chiral assembly of PF with branched side chain and investigate the chiral assembly mechanism, PF2/6 and PF8 (Scheme 1) were taken as examples to be aggregated in a binary solvent system. In the binary solvent system, chiral limonene $(1 R / 1 S)$ was used as a good solvent and chiral inducer, and ethanol (EtOH) was employed as a poor solvent.

It is well known that the volume fraction of the poor solvent obviously affects the chiral assembly behaviour in a multisolvent system..$^{20,22,25,26}$ Higher or lower content of the poor solvent will result in a weak assembly and precipitation of the polymer from the multi-solvent system. UV-visible (UV-vis), photoluminescence (PL) and circular dichroism (CD) spectroscopy were employed to investigate the chiroptical properties of $\mathrm{PF}$ aggregates in different volume fractions of limonene and ethanol. When the PF chains were completely dissolved in neat limonene at the molecular level, a typical absorption peak for $\alpha$ phase was observed at around $380 \mathrm{~nm}$ for both PF2/6 and PF8 in the UV-vis spectra (Fig. 1). In this case, the planar angle between adjacent fluorene units in the polymer main chain is about $135^{\circ}$. With the gradual increase in the proportion of the poor solvent (EtOH), the UV-vis absorption intensity of PF2/6 decreased step by step, indicating the formation of aggregates. For PF8, it was remarkable that an obvious absorption shoulder peak appeared near $435 \mathrm{~nm}$, and the absorption intensity increased gradually with the increase in the poor solvent fraction. This is a characteristic absorption of a conformational intramolecular isomer ( $\beta$ phase), in which the intrachain torsion angle is about $165^{\circ}$. Studies have shown that the formation mechanism of $\beta$ phase is divided into two steps. ${ }^{46}$ The first step is the energy storage process. The poor solvent reduces the interaction between the PF chains and the good solvent, and increases the force between the PF chains, which causes the formation of aggregates. A further increase in the poor solvent fraction results in strong aggregation, making the PF backbone planar. Compared with the $\alpha$ phase, the more planar $\beta$ phase of $\mathrm{PF}$ increases the effective conjugation length
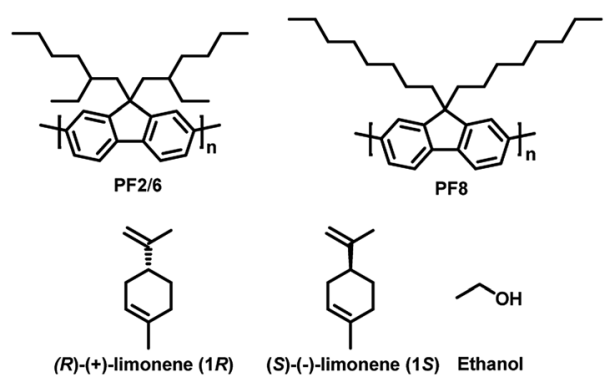

Scheme 1 Chemical structures of poly(9,9-bis(2-ethylhexyl)-fluorene) (PF2/6), poly(9,9-dioctyl-fluorene) (PF8), limonene (1R and $1 S)$ and achiral poor solvent.
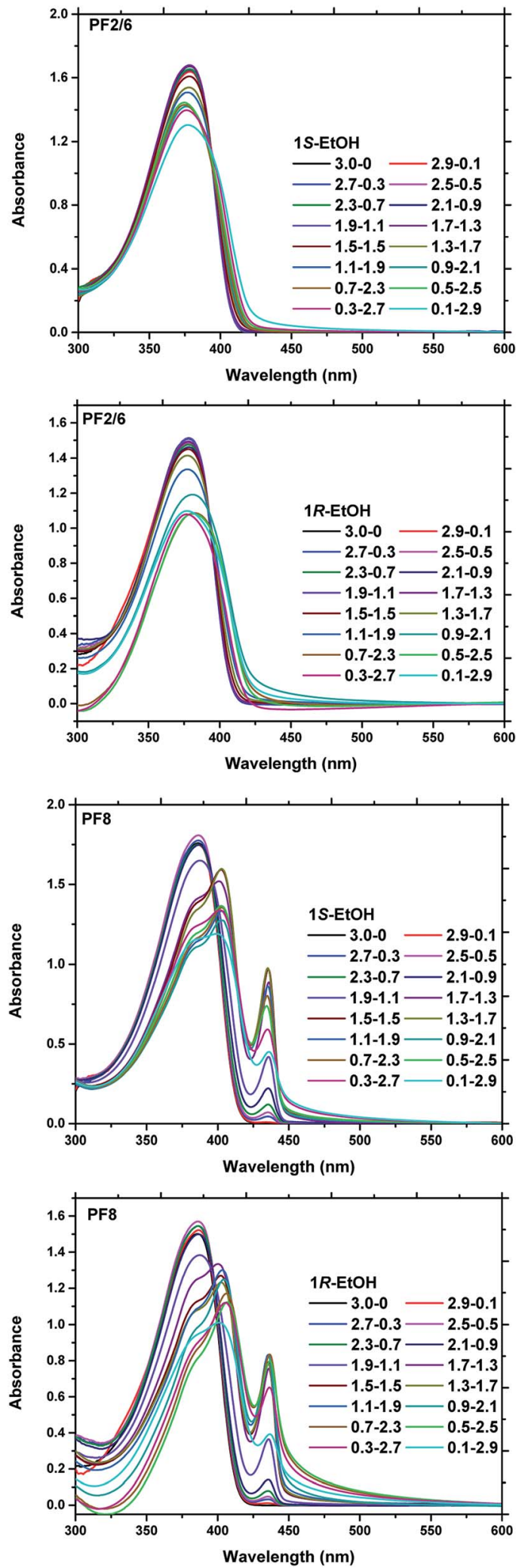

Fig. 1 UV-vis spectra of PF2/6 and PF8 solutions and aggregates in binary solvent with different $1 S / \mathrm{EtOH}$ and $1 R / \mathrm{EtOH}$ volume fractions. The concentration of polymer repeating units was $5 \times 10^{-5} \mathrm{~mol} \mathrm{~L}^{-1}$.

of polymer chains, ${ }^{47}$ which is beneficial to the $\pi-\pi$ stacking between the PF backbones. It is found that as the volume of EtOH increases, the $\beta$ phase content tends to gradually increase 
and finally reach about $40 \%$ (Fig. S2 $\dagger$ ), which is consistent with the saturation value as recently reported.$^{49}$ Not only that, when the volume ratio of limonene to EtOH is $1.7 / 1.3$, the UV-vis absorption peak of PF8 changes significantly from $386 \mathrm{~nm}$ to $403 \mathrm{~nm}$ with a $17 \mathrm{~nm}$ red shift. However, the UV-vis absorption shows a very different behaviour for PF2/6. During the process of forming the aggregates, the absorption peak (at $380 \mathrm{~nm}$ ) of PF $2 / 6$ only changes in absorption intensity without an obvious red shift. This phenomenon indicates that the phase of PF2/6 main chain is still the $\alpha$ phase and has not been converted into the $\beta$ phase during the assembly process.

The photoluminescence (PL) emission spectra of the polymer solution and the aggregate solution were investigated under an excitation of $370 \mathrm{~nm}$ as presented in Fig. 2. It can be seen from Fig. 2 that the polymer solutions of both PF2/6 and PF8 exhibit typical emission peaks of the $\alpha$ phase, that is, three vibrational spectral bands: $0-0^{\prime}(411-416 \mathrm{~nm}), 0-1^{\prime}(432-440$ $\mathrm{nm}), 0-2^{\prime}(464-466 \mathrm{~nm}){ }^{44}$ The information expressed by the PL spectra is consistent with that by the UV-vis spectra. From the PL spectra of the aggregate solution of PFs, we can see that with the increase in the poor solvent fraction, the PL emission peak of PF2/6 maintains the same shape but changes slightly from $411 \mathrm{~nm}$ to $418 \mathrm{~nm}$ with a $7 \mathrm{~nm}$ red shift. Its intensity shows a trend of slight increase first and then a sharp decrease (Fig. S3†). The possible reason for this phenomenon is that the addition of a poor solvent (ethanol) in the initial stage increases the polarity of the cosolvent, which causes an increase in the PL emission intensity. When the binary solvent ratio reached $1.7 /$ 1.3 (limonene/EtOH, v/v), the polymer chain began to aggregate (as indicated by the DLS data, Fig. S4 $\dagger$ ). On account of the aggregation caused quenching (ACQ), the PL emission intensity of PF2/6 gradually decreased. These results indicated that the $\mathrm{PF} 2 / 6$ chain had the same conformation as the $\alpha$ phase in the solution and aggregate state. Nevertheless the PL emission peak of PF8 at $416 \mathrm{~nm}$ gradually weakened and finally disappeared with the progress of aggregation. Meanwhile, the emission peak at $440 \mathrm{~nm}$ and $466 \mathrm{~nm}$ gradually increased, and a new emission peak appeared at $498 \mathrm{~nm}$. These features of the change in emission peaks indicate that the conformation of PF8 changes from $\alpha$ phase to $\beta$ phase, and the dihedral angle between the adjacent repeat units of PF8 changes from $135^{\circ}$ to $165^{\circ}$ with the occurrence of aggregation.

The above spectral data indicated that PF8 had a conformation conversion during the process of aggregation, while PF2/6 did not. Our previous study indicated that the supramolecular chiral aggregation of PF8 could be successfully induced by neat limonene at low temperature $\left(-10^{\circ} \mathrm{C}\right.$ to $\left.-30^{\circ} \mathrm{C}\right)$; however, $\mathrm{PF} 2 /$ 6 could not perform chiral assembly even at a much lower temperature $\left(-80^{\circ} \mathrm{C}\right) .{ }^{44}$ Then, the chiral assembly behaviour of PF2/6 and PF8 in the current binary solvent system (limonene/ $\mathrm{EtOH}$ ) was further studied. More interestingly, the CD spectra of $\mathrm{PF} 2 / 6$ aggregates in $1 R$ or $1 S / \mathrm{EtOH}$ showed a positive/negative signal with nearly ideal mirror-image in the proper volume fraction of each solvent (Fig. 3(a)). This is the first report on the chiral induction in achiral PF with branched side alkyl chains by chiral solvation. The maximum CD amplitude of PF2/6 aggregates was affected by the limonene/EtOH volume
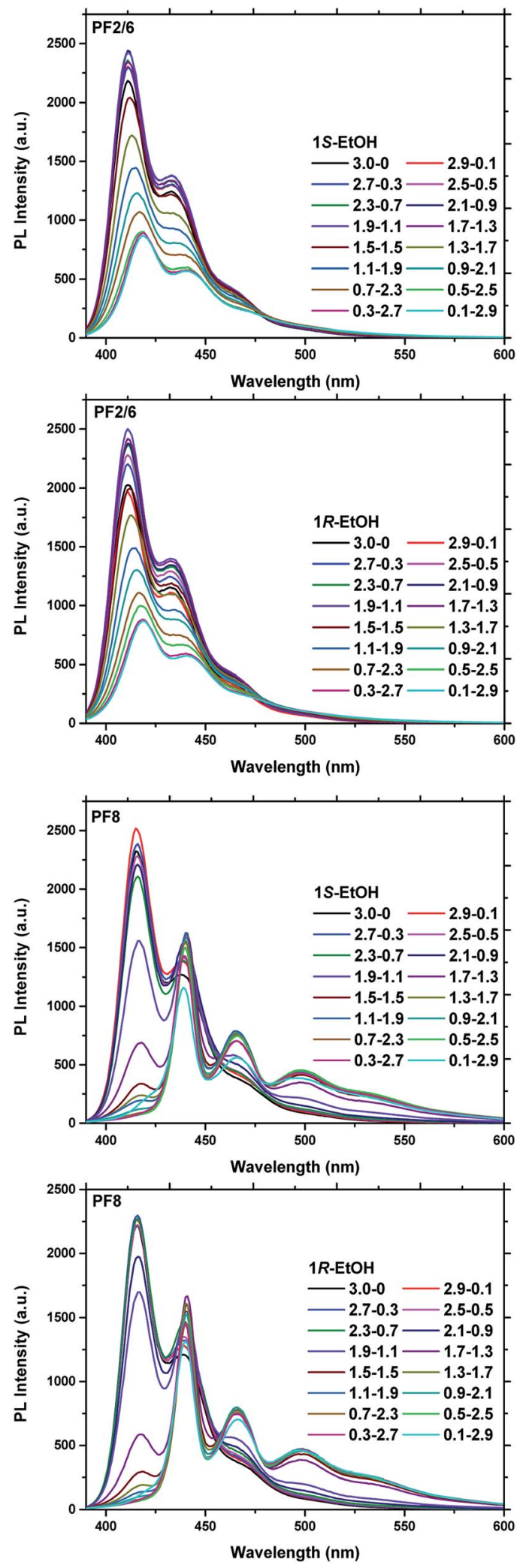

Fig. 2 PL spectra of PF2/6 and PF8 solutions and aggregates in binary solvent with different $1 \mathrm{~S} / \mathrm{EtOH}$ or $1 R / \mathrm{EtOH}$ volume fractions. The concentration of polymer repeating units was $5 \times 10^{-5} \mathrm{~mol} \mathrm{~L}^{-1}$.

fraction. The CD signal appeared and gradually increased when the volume fraction of limonene/EtOH changed from 1.7/1.3 to $0.1 / 2.9$. This change is attributed to the weak aggregation when 

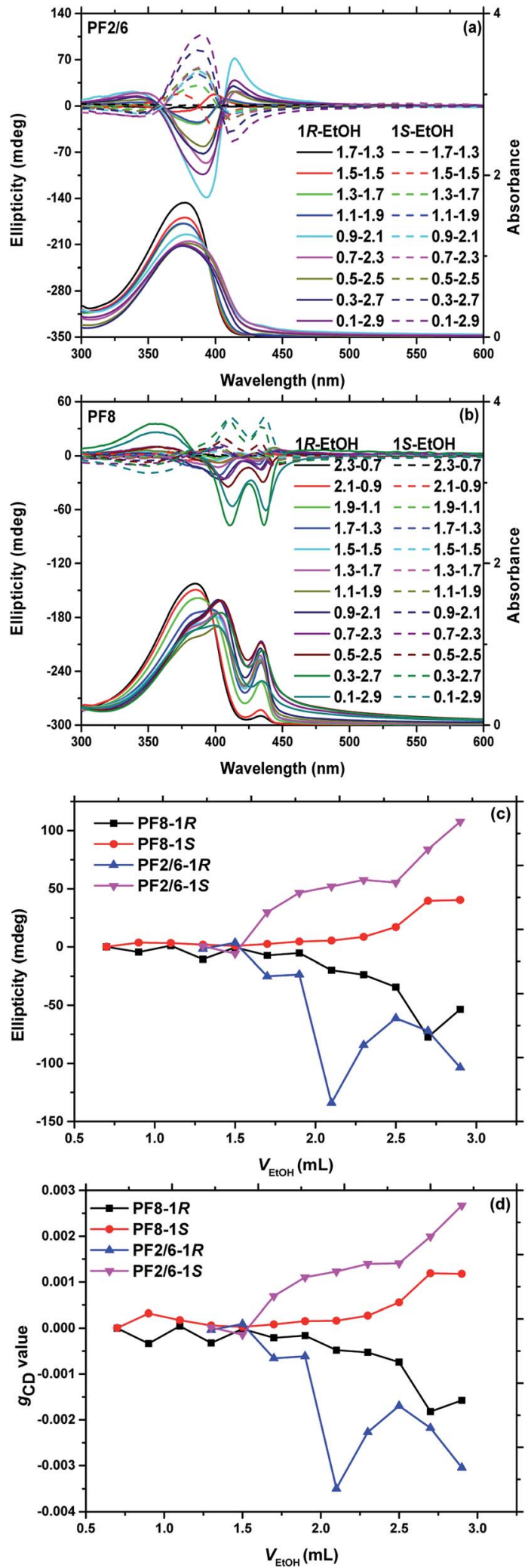

Fig. 3 UV-vis absorption and CD spectra of PF2/6 (a) and PF8 (b) aggregates in binary solvent with different (1S or $1 R$ )/EtOH volume fractions. The maximum $C D$ (c) and $g_{C D}$ (d) values of PF aggregates at around $390 \mathrm{~nm}$ (PF2/6) and $410 \mathrm{~nm}$ (PF8) in binary solvent with different (1S or $1 R$ )/EtOH volume fractions. The concentration of polymer repeating units was $5 \times 10^{-5} \mathrm{~mol} \mathrm{~L}^{-1}$. the poor solvent content is relatively lower, as clarified in our previous studies. ${ }^{32}$ However, it is abnormal that the CD intensity increased sharply with the volume fraction of $0.9 / 2.1$ and $0.7 / 2.3$ $(1 R / \mathrm{EtOH})$. The reason for it is unclear so far, which may be due to the polarity or refractive index change with different volume fractions of the cosolvents, as reported previously. ${ }^{50}$ Meanwhile PF2/6 aggregates exhibited three CD signal peaks, typically for the $\alpha$ phase, at the first (420-417 nm), second (377-391 nm), and third $(341 \mathrm{~nm})$ cotton bands, showing gradual red shifts with the increase in the poor solvent fraction. A possible reason is the accumulation of polymer chains in the aggregates.

As compared to PF2/6, a similar dependence of CD intensity of PF8 aggregates on the volume fraction of limonene/EtOH was observed (Fig. 3(b)), i.e. an increase with the gradual increase in the poor solvent volume fraction. Nevertheless PF8 exhibited four CD signal peaks at the first (around $445 \mathrm{~nm}$ ), second (around $433 \mathrm{~nm}$ ), third (around $405 \mathrm{~nm}$ ), and fourth (around $356 \mathrm{~nm}$ ) cotton band with the proper volume fraction of limonene/EtOH. The cotton bands at around $445 \mathrm{~nm}$ and $433 \mathrm{~nm}$ are typically derived from the chiral $\beta$ phase, which is due to the helical $\pi-\pi$ stacked structures of fully extended anticoplanar PF8 chains. It was noticeable that the positive/negative signal of PF2/6 was not centred on the maximum absorption position, in contrast with that of PF8, which may be a proof that interchain exciton coupling is not the origin of $\mathrm{CD}$ in PF2/6 (intrachain helicity) but it is so in PF8 (interchain helicilty).

The dependence of the maximum CD values and dissymmetry factors in absorption, $g_{\mathrm{CD}}$, on limonene/EtOH volume fraction are summarized in Fig. 3(c) and (d), respectively. As presented in Fig. 3, the $g_{\mathrm{CD}}$ and the maximum CD values of PF2/ 6 show an increasing trend with the enhancement of the poor solvent volume ratio, except in the case of 0.9/2.1 and 0.7/2.3 $(1 R / \mathrm{EtOH})$. It is noted that the $\beta$ phase of PF8 appears at $2.7 /$ 0.3 (limonene/EtOH), but the CD signal is observed at 2.1/0.9 (limonene/EtOH) with the $\beta$ phase content of $10 \%$. It means that chiral supramolecular aggregation only occurs when a certain extent of accumulation of $\beta$ phase is reached. By comparing the $g_{\mathrm{CD}}$ and $\beta$ phase content of PF8, some interesting results can be found. The $\beta$ phase begins to appear at a ratio of

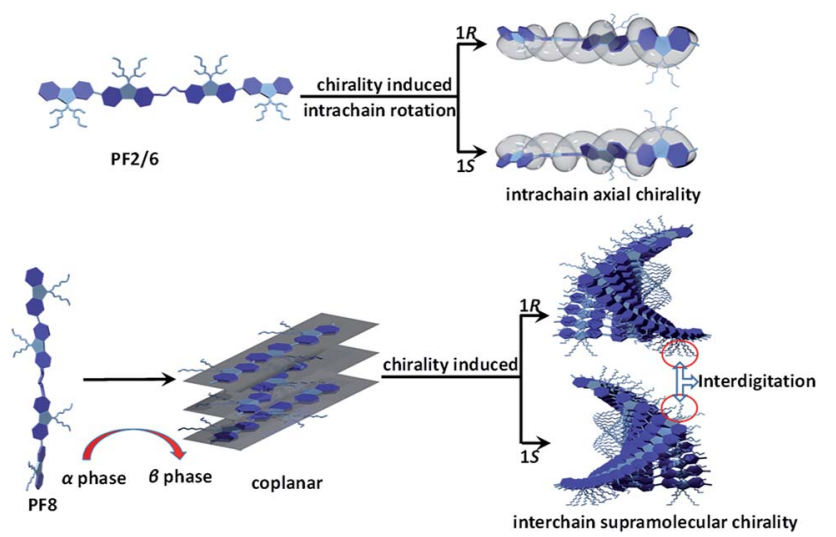

Scheme 2 The proposed chiral assembly mechanism of PF2/6 and PF8. 
2.7/0.3 (limonene/EtOH, v/v), and the content increases sharply to approximately $35 \%$ at $1.7 / 1.3$. The $g_{\mathrm{CD}}$ data shows that PF8 is induced to demonstrate chirality at a ratio of 2.1/0.9 (limonene/ EtOH, v/v) and the $g_{\mathrm{CD}}$ data begins to increase gradually at a ratio of 1.7/1.3. These results indicate that it is very important for PF8 to undergo a conformation transition from $\alpha$ phase to $\beta$ phase during the process of chiral induction.

Considering the above results, it can be concluded that the mechanism of chiral self-assembly of PF2/6 and that of PF8 are quite different as proposed in Scheme 2. PF2/6 may assume an exact alternately twisted structure in the optically inactive state. Due to the free rotation about the single bonds between the adjacent fluorene rings, the $\mathrm{PF} 2 / 6$ main chain can switch between left and right helix. PF2/6 retains a single polymer chain in the poor solvent, ${ }^{\mathbf{5 1}}$ and no conformation transition occurs during the process of chiral induction. The main chain of PF2/6 can rotate to form the preferred twist with the aid of chiral limonene without a conformation change, which results in excess of P- or M-helix. PF8 undergoes a conformational transformation during the process of aggregation, ${ }^{27}$ resulting in
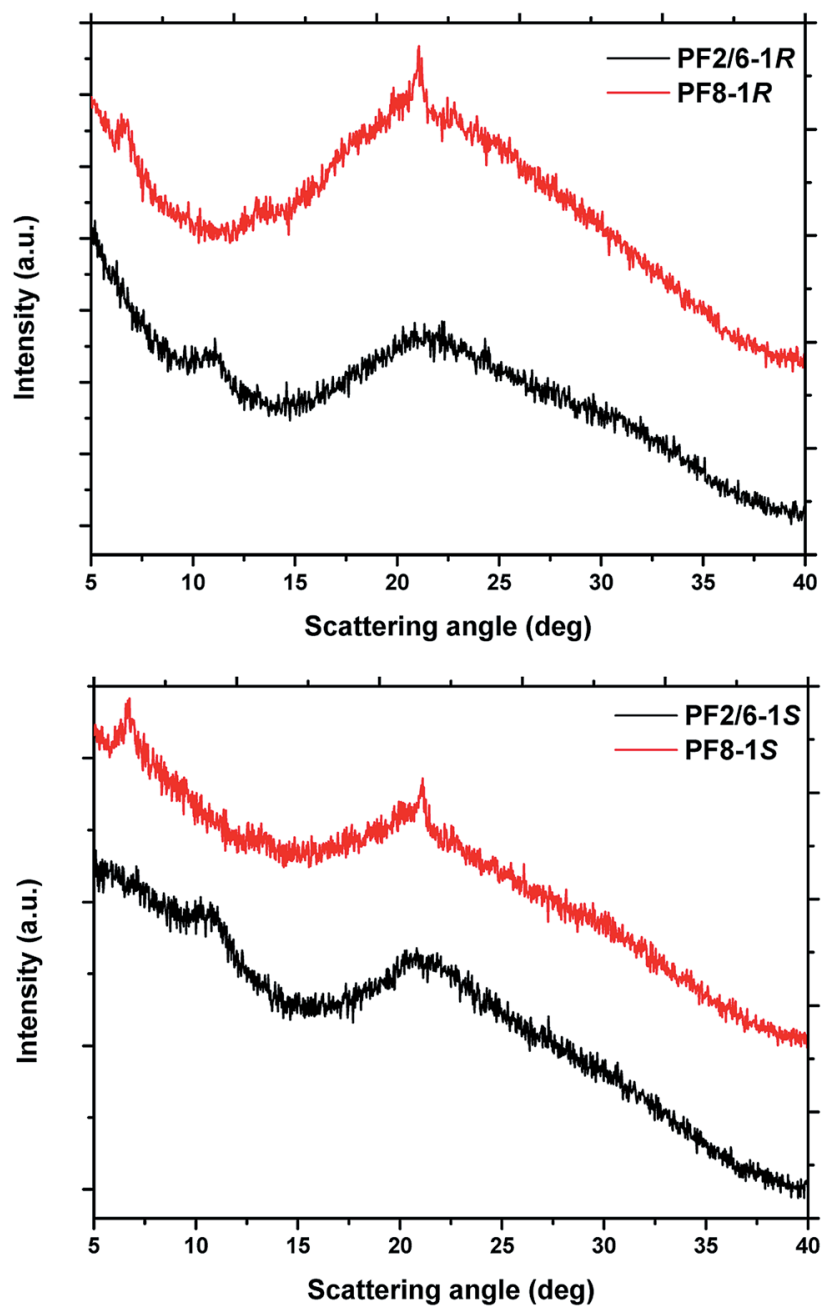

Fig. 4 WAXD profiles of PF2/6 and PF8 films. The PF films were cast from freshly prepared aggregate solutions of $10 \mathrm{mg} \mathrm{mL}^{-1} \mathrm{PFs}$ in binary solvent, and the volume fraction of (1S or $1 R) / \mathrm{EtOH}$ was 1.0/2.0. a more planar $\beta$ phase, which is to the benefit of the $\pi-\pi$ stacking of PF8 main chain. Meanwhile, the interdigitation of the side alkyl chains is possible in PF8 but it may be prevented in PF $2 / 6$ by the bulk of the branched alkyl chains, which also helps the PF8 chain to form the supramolecular structure. With the help of intermolecular chiral $\mathrm{CH}-\pi$ interactions between the alkyl chains and chiral limonene and intermolecular chiral $\pi-\pi$ interactions among main chains, the PF8 chain selfassembles into a helical supramolecular structure.

In order to obtain further information on PF2/6 and PF8 aggregates, WAXD and TEM results were acquired as shown in Fig. 4 and S6. $\uparrow$ The WAXD profile of PF8 exhibited two obvious peaks at around $2 \theta=7^{\circ}$ and $21^{\circ}$. The first peak is the characteristic peak of $\beta$ phase. The $21^{\circ}$ peak may be the common characteristic of many polymers substituted by side chains and indicates the degree of disorder in the packing of the alkyl side chains of PF8. ${ }^{52}$ The WAXD profile of PF2/6 exhibited a broad peak at $2 \theta=11^{\circ}$, which may correspond to the hexagonal phase and a higher degree of interchain disorder. ${ }^{53}$ The TEM images
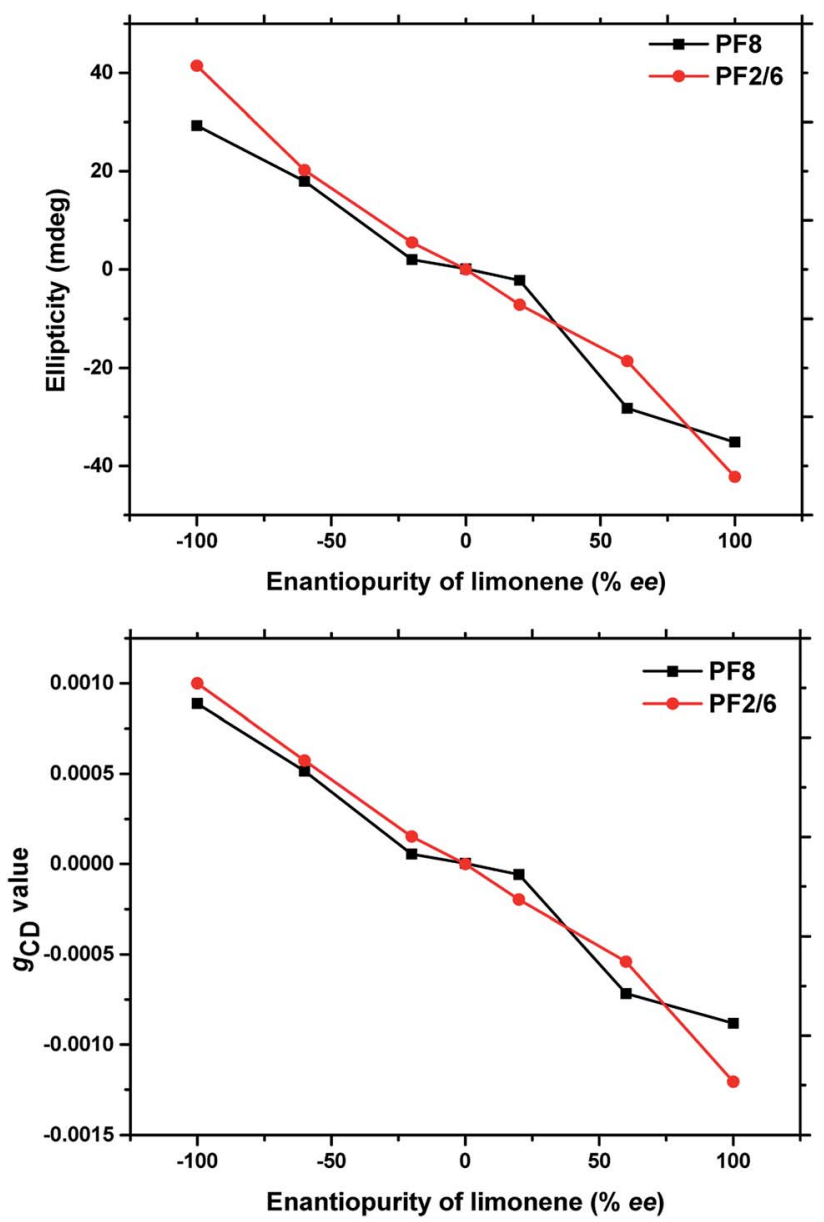

Fig. 5 Plots of maximum $C D$ and $g_{C D}$ values of $P F$ aggregates at around $390 \mathrm{~nm}$ (PF2/6) and $410 \mathrm{~nm}$ (PF8) in binary solvent measured with different enantiopurity values of limonene. The volume fraction of $(1 S$ or $1 R$ )/EtOH was $0.3 / 2.7$. The concentration of polymer repeating units was $5 \times 10^{-5} \mathrm{~mol} \mathrm{~L}^{-1}$. The maximum $\mathrm{CD}$ and $g_{\mathrm{CD}}$ values were taken and calculated from Fig. S5. $\uparrow$ 
showed that both PF2/6 and PF8 aggregates had nanofiber morphology. However, the helical sense was difficult to be observed clearly.

In previous reports, the chirality of linear $\pi$-conjugate polymer aggregates was linearly controlled by the enantiomeric purity of the chiral solvent. ${ }^{20,22}$ To investigate whether PF2/6 and PF8 follow this rule, the effect of the enantiomeric purity of chiral limonene on the maximum value of $\mathrm{CD}$ and the $g_{\mathrm{CD}}$ values was studied, as presented in Fig. 5. The volume fraction of binary solvents was maintained as $0.3 / 2.7$ for both PF2/6 and PF8, just changing the chiral solvent $(1 R / 1 S)$ ratio. The maximum value of $\mathrm{CD}$ and the $g_{\mathrm{CD}}$ values were almost linearly controlled by the respective molar ratio of enantiomers $(1 R / 1 S)$, indicating that no obvious chiral amplification occurred in the current system.

\section{Conclusions}

In summary, we demonstrated that polyfluorene (PF) derivatives with branched (PF2/6) and linear (PF8) side alkyl chains performed chiral assembly with different mechanisms in a binary solvent system for the first time. The chirality of PF2/6 aggregates is from the axial chirality of the polymer chain with the $\alpha$ phase conformation; however, that of PF8 is derived from the chiral supramolecular assembly of PF8 main chains along with the more coplanar $\beta$ phase conformation, induced by chiral limonene solvation. The first successful chirality induction in PF derivatives with branched side chains not only enriches the construction of PF-based chiral materials from achiral counterparts, but also gives further insight into the chiral assembly mechanism of PFs with different topological side chain structures.

\section{Conflicts of interest}

There are no conflicts to declare.

\section{Acknowledgements}

The authors are grateful for the financial support from the National Nature Science Foundation of China (21971180), Nature Science Key Basic Research of Jiangsu Province for Higher Education (no. 19KJA360006), the Priority Academic Program Development (PAPD) of Jiangsu Higher Education Institutions and the Program of Innovative Research Team of Soochow University.

\section{Notes and references}

1 M. H. Liu, L. Zhang and T. Y. Wang, Chem. Rev., 2015, 115, 7304-7397.

2 S. J. George, Z. Tomovic, A. P. H. J. Schenning and E. W. Meijer, Chem. Commun., 2011, 47, 3451-3453.

3 H. Z. Tang, M. Fujiki and T. Sato, Macromolecules, 2002, 35, 6439-6445.

4 H. Z. Tang, M. Fujiki and M. Motonaga, Polymer, 2002, 43, 6213-6220.
5 C. Kulkarni, R. Munirathinam and S. J. George, Chem.-Eur. J., 2013, 19, 11270-11278.

6 J. Kim, J. Lee, W. Y. Kim, H. Kim, S. Lee, H. C. Lee, Y. S. Lee, M. Seo and S. Y. Kim, Nat. Commun., 2015, 6, 6959.

7 L. B. Wang, L. Yin, W. Zhang, X. L. Zhu and M. Fujiki, J. Am. Chem. Soc., 2017, 139, 13218-13226.

8 T. Mori, M. Kyotani and K. Akagi, Macromolecules, 2008, 41, 607-613.

9 K. Akagi, Chem. Rev., 2009, 109, 5354-5401.

10 P. Dellaportas, R. G. Jones and S. J. Holder, Macromol. Rapid Commun., 2002, 23, 99-103.

11 H. Nakashima, J. R. Koe, K. Torimitsu and M. Fujiki, J. Am. Chem. Soc., 2001, 123, 4847-4848.

12 M. M. Green, C. Khatri and N. C. Peterson, J. Am. Chem. Soc., 1993, 115, 4941-4942.

13 T. F. Miao, L. Yin, X. X. Cheng, Y. Zhao, W. J. Hou, W. Zhang and X. L. Zhu, Polymers, 2018, 10, 612.

14 J. Wang, G. Yang, H. Jiang, G. Zou and Q. J. Zhang, Soft Matter, 2013, 9, 9785-9791.

15 Z. C. Shen, T. Y. Wang, L. Shi, Z. Y. Tang and M. H. Liu, Chem. Sci., 2015, 6, 4267-4272.

16 J. M. Ribó, J. Crusats, F. Sagués, J. Claret and R. Rubires, Science, 2001, 292, 2063-2066.

17 P. L. Chen, X. G. Ma, K. M. Hu, Y. L. Rong and M. H. Liu, Chem.-Eur. J., 2011, 17, 12108-12114.

18 X. X. Cheng, T. F. Miao, L. Yin, H. L. Chen, M. Liu, W. Zhang and X. L. Zhu, J. Funct. Polym., 2019, DOI: 10.14133/ j.cnki.1008-9357.20190329001.

19 B. Bosnich, J. Am. Chem. Soc., 1967, 89, 6143-6148.

20 W. Zhang, K. Yoshida, M. Fujiki and X. L. Zhu, Macromolecules, 2011, 44, 5105-5111.

21 L. B. Wang, N. Suzuki, J. F. Liu, T. Matsuda, N. A. A. Rahim, W. Zhang, M. Fujiki, Z. B. Zhang, N. C. Zhou and X. L. Zhu, Polym. Chem., 2014, 5, 5920-5927.

22 S. Q. Jiang, Y. Zhao, L. Wang, L. B. Yin, Z. B. Zhang, J. Zhu, W. Zhang and X. L. Zhu, Polym. Chem., 2015, 6, 4230-4239.

23 V. Stepanenko, X. Q. Li, J. Gershberg and F. Würthner, Chem.-Eur. J., 2013, 19, 4176-4183.

24 A. M. Buono, I. Immediata, P. Rizzo and G. Guerra, J. Am. Chem. Soc., 2007, 129, 10992-10993.

25 Y. Kawagoe, M. Fujiki and Y. Nakano, New J. Chem., 2010, 34, 637-647.

26 Y. Nakano, Y. Liu and M. Fujiki, Polym. Chem., 2010, 1, 460469.

27 Y. Zhao, N. A. Abdul Rahim, Y. J. Xia, M. Fujiki, B. Song, Z. B. Zhang, W. Zhang and X. L. Zhu, Macromolecules, 2016, 49, 3214-3221.

28 J. J. Liu, Y. Zhao, H. L. Chen, Z. B. Zhang, W. Zhang and X. L. Zhu, React. Funct. Polym., 2017, 121, 76-81.

29 H. Kim, Y. J. Jin, B. S. I. Kim, T. Aoki and G. Kwak, Macromolecules, 2015, 48, 4754-4757.

30 J. F. Liu, J. Zhang, S. S. Zhang, N. Suzuki, M. Fujiki, L. B. Wang, L. Li, W. Zhang, N. C. Zhou and X. L. Zhu, Polym. Chem., 2014, 5, 784-791.

31 L. Yin, Y. Zhao, S. Q. Jiang, L. B. Wang, Z. B. Zhang, J. Zhu, W. Zhang and X. L. Zhu, Polym. Chem., 2015, 6, 7045-7052. 
32 L. Yin, Y. Zhao, M. Liu, N. C. Zhou, W. Zhang and X. L. Zhu, Polym. Chem., 2017, 8, 1906-1913.

33 L. Yin, M. Liu, Y. Zhao, S. S. Zhang, W. Zhang, Z. B. Zhang and X. L. Zhu, Polym. Chem., 2018, 9, 769-776.

34 E. Peeters, M. P. T. Christiaans, R. A. J. Janssen, H. F. M. Schoo, H. P. J. M. Dekkers and E. W. Meijer, J. Am. Chem. Soc., 1997, 119, 9909-9910.

35 K. Watanabe, I. Osaka, S. Yorozuya and K. Akagi, Chem. Mater., 2012, 24, 1011-1024.

36 M. Oda, H.-G. Nothofer, G. Lieser, U. Scherf, S. C. J. Meskers and D. Neher, Adv. Mater., 2000, 12, 362-365.

37 M. Oda, H.-G. Nothofer, U. Scherf, V. Šunjic, D. Richter, W. Regenstein and D. Neher, Macromolecules, 2002, 35, 6792-6798.

38 B. Liu and G. C. Bazan, J. Am. Chem. Soc., 2006, 128, 11881196.

39 H. Goto and E. Yashima, J. Am. Chem. Soc., 2002, 124, 79437949.

40 M. R. Craig, P. Jonkheijm, S. C. J. Meskers, A. P. H. J. Schenning and E. W. Meijer, Adv. Mater., 2003, 15, 1435-1438.

41 R. Abbel, A. P. H. J. Schenning and E. W. Meijer, Macromolecules, 2008, 41, 7497-7504.

42 Y. Wang, T. Sakamoto and T. Nakano, Chem. Commun., 2012, 48, 1871-1873.
43 Y. Wang, T. Harada, L. Q. Phuong, Y. Kanemitsu and T. Nakano, Macromolecules, 2018, 51, 6865-6877.

44 Y. Zhao, H. L. Chen, L. Yin, X. X. Cheng, W. Zhang and X. L. Zhu, Polym. Chem., 2018, 9, 2295-2301.

45 N. A. A. Rahim and M. Fujiki, Polym. Chem., 2016, 7, 46184629.

46 L. Huang, T. Li, B. Liu, L. L. Zhang, Z. M. Bai, X. N. Li, X. N. Huang and D. Lu, Soft Matter, 2015, 11, 2627-2638.

47 M. Grell, D. D. C. Bradley, G. Ungar, J. Hill and K. S. Whitehead, Macromolecules, 1999, 32, 5810-5817.

48 C. Zanlorenzi, B. Nowacki and L. Akcelrud, J. Mater. Chem. C, 2019, 7, 6161-6168.

49 X. N. Li, Z. M. Bai, B. Liu, T. Li and D. Lu, J. Phys. Chem. C, 2017, 121, 14443-14450.

50 M. Fujiki, K. Yoshida, N. Suzuki, J. Zhang, W. Zhang and X. L. Zhu, RSC Adv., 2013, 3, 5213-5219.

51 M. Knaapila, V. M. Garamus, F. B. Dias, L. Almasy, F. Galbrecht, A. Charas, J. Morgado, H. D. Burrows, U. Scherf and A. P. Monkman, Macromolecules, 2006, 39, 6505-6512.

52 S. K. Lee, T. Ahn, J. H. Park, Y. K. Jung, D. S. Chung, C. E. Park and H. K. Shim, J. Mater. Chem., 2009, 19, 70627069.

53 B. Tanto, S. Guha, C. M. Martin, U. Scherf and M. J. Winokur, Macromolecules, 2004, 37, 9438-9448. 